\title{
Marine biotechnology: Emerging opportunities and future perspectives
}

Symposium held at Sultan Qaboos University, November 12-13th 2013.

Stephen Goddard

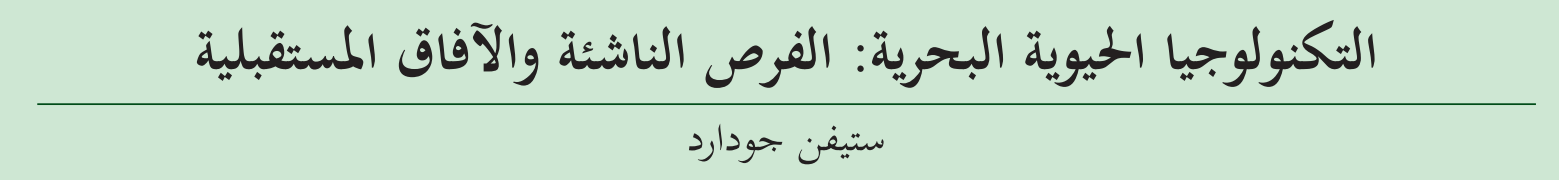

$\mathrm{T}$ The rapid growth of genetic, cellular and molecular technologies is enabling scientists to explore and develop marine resources for widespread applications in the food, medical, pharmaceutical, environmental and energy industries. Marine biotechnology products and services were estimated at 2.8 billion euros in 2010, with a cumulative annual growth rate of 4-5\% (Marine Board-European Science Foundation, 2010) The Sultanate of Oman occupies a strategic geographical position and has a coastline in excess of $3000 \mathrm{~km}$ with the Arabian Sea located to the south and the Sea of Oman and Arabian Gulf to the north. These oceans have rich biodiversity and potential resources which we are only beginning to explore. Based on its marine resources, coupled with a rapidly-growing educational and research infrastructure, the Sultanate of Oman is well positioned to take advantage of the commercial opportunities presented by marine biotechnology.

In recognition of potential development an international symposium was organized by the Center of Excellence in Marine Biotechnology, Sultan Qaboos University. One hundred and forty eight delegates attended the meeting, representing 15 countries. In planning the symposium three major themes were identified viz. Marine Biofouling and its Prevention, Fisheries and Aqua- culture Biotechnology and Marine Biodiversity and Natural Products. The topics were selected on the basis of current and planned research activity in Oman and elsewhere in the GCC region. Three keynote addresses were presented, 23 oral presentations made and a poster exhibition held. A final session reviewed national and regional issues and the delegates agreed formally on a number of future actions. Full details of the symposium and the results and analysis of a post-symposium survey on the future development of marine biotechnology are given in Goddard et al. (2015).

The symposium was supported logistically and financially by Sultan Qaboos University, The Research Council, and the Ministry of Agriculture and Fisheries Wealth, Oman.

\section{References}

Goddard, S., M. Delghandi, S. Dobretsov, H. Al-Oufi, S. Al-Habsi, and J.G. Burgess 2015. The first GCC Marine Biotechnology Symposium: Emerging Opportunities and Future Perspectives. Marine Biotechnology 17: 285-289.

Marine Board-European Science Foundation, 2010. Marine Biotechnology: a new vision and Strategy for $\mathrm{Eu}$ rope. Position paper 15 . 